\title{
Exploration d'un système interfacique et de ses dynamiques : application au tourisme de réunions et de congrès
}

\author{
S. Christofle ${ }^{a}$
}

\begin{abstract}
Géographe, Université Nice Sophia Antipolis-Institut Ulysse, U.F.R. Espaces et Cultures - Département Géographie, Aménagement et Environnement durable, UMR ESPACE $n^{\circ} 7300$ - Equipe G.V.E, Tourisme, Campus Carlone, 98 boulevard Herriot, BP. 3209, 06204 Nice Cedex 03, France
\end{abstract}

\begin{abstract}
Résumé. Le tourisme de réunions et de congrès fait se rassembler en un lieu nodal, durant au moins deux jours, des personnes issues d'horizons géographiques variés mais aux intérêts communs. Le système interfacique congressuel se structure autour de la réunion, interface immatérielle, qui prend place dans le palais des congrès, interface matérielle, et met en contact différents acteurs : congressistes, organisateurs, prestataires de services, collectivités territoriales. Ce système ouvert, localisé, s'articule autour de trois grands types d'interfaces, intra-réticulaires, inter-réseaux et réseaux-territoires dans le cadre de stratégies économiques, de destination et de développement territorial. Quatre grands effets sont induits : la diffusion et l'appropriation d'une information neuve, l'accélération de l'innovation et de l'expérimentation, la création d'une expérience communautaire, le développement de la valeur.
\end{abstract}

Tout d'abord : qu'est-ce que le tourisme?

En 2000, une définition institutionnelle a été mise en place par l'Organisation Mondiale du Tourisme (OMT) et la Commission statistique de l'Organisation des Nations Unies $(\mathrm{ONU})^{1}$, reprise en France par l'INSEE (Institut National de la Statistique et des Etudes économiques) et le Ministère de l'Economie, des Finances et de l'Industrie (DGCIS). Le tourisme correspondrait donc à «l'ensemble des activités déployées par les personnes au cours de leurs voyages et séjours dans des lieux situés en dehors de leur environnement habituel, pour une période consécutive qui ne dépasse pas une année, à des fins de loisirs, pour affaires et autres motifs non liés à l'exercice d'une activité rémunérée dans le lieu visité ». Cette « définition» se révèle extensive et englobant de très vastes champs potentiels d'étude tant en termes de thématique («pratiques» ou «activités» caractérisant le déplacement touristique : tourisme de découverte, tourisme sportif, voire tourisme médical, par exemple) que de localisation (tourisme de montagne, tourisme urbain...). Apparaît alors le besoin d'une assise théorique plus solide qui n'est pas encore déterminée. En effet, si la définition institutionnelle est discutée, critiquée, amendée par la communauté scientifique (par exemple Boyer, 2002, Stock, 2003, Meyer, 2005) aucune conceptualisation ne fait l'objet d'un véritable consensus entre chercheurs. Dans ce cadre aux contours un peu flous, mais porteur de dynamisme, se pose alors la question :

\footnotetext{
a e-mail : Christof@unice.fr

${ }^{1}$ Nations Unies et Organisation Mondiale du Tourisme, Compte satellite du tourisme : recommandation concernant le cadre conceptuel, 2001.
}

Qu'est-ce que le tourisme de réunions et de congrès -TRC?

La définition suivante va être utilisée dans ce travail : «Le TRC rassemble physiquement pendant plusieurs jours des personnes en provenance d'horizons géographiques variés qui partagent un ou des intérêts communs (d'ordre économique, scientifique, associatif, de loisirs, sportif, religieux ou politique). La manifestation revêt prioritairement, mais pas exclusivement, un caractère professionnel ou socio-professionnel. La mobilité qui lui est associée est contrainte ou volontaire et s'accompagne souvent de pratiques touristiques de loisirs » (Christofle, Fabry, Morschel, 2010a) La typologie (Fig. 1) qui suit (Christofle, Fabry, Morschel, 2010a) présente les types majeurs de meetings constituant le TRC.

\section{Tourisme de réunions et de congrès et système interfacique congressuel}

Le tourisme de réunions et de congrès fait donc se rencontrer en face-à-face, en un lieu de rassemblement, des participants de toutes origines géographiques pour une réunion qui dure a minima deux jours. Le tourisme de réunions et de congrès est une activité hybride, partagée entre temps de réunion (sessions) et temps de loisirs. L'objectif de déplacement peut avoir une finalité professionnelle ou associative, et les congressistes, ainsi que leurs accompagnants, s'adonnent à des activités diversifiées de découverte, de loisirs, de rencontre, d'échange et d'interaction, brisant leur routine quotidienne (Davidson, 2003, Christofle, 2011). L'activité congressuelle se localise dans des espaces variés mais tend à se concentrer dans les grands lieux d'ancrage de la mondialisation tant économique que touristique, culturelle, 


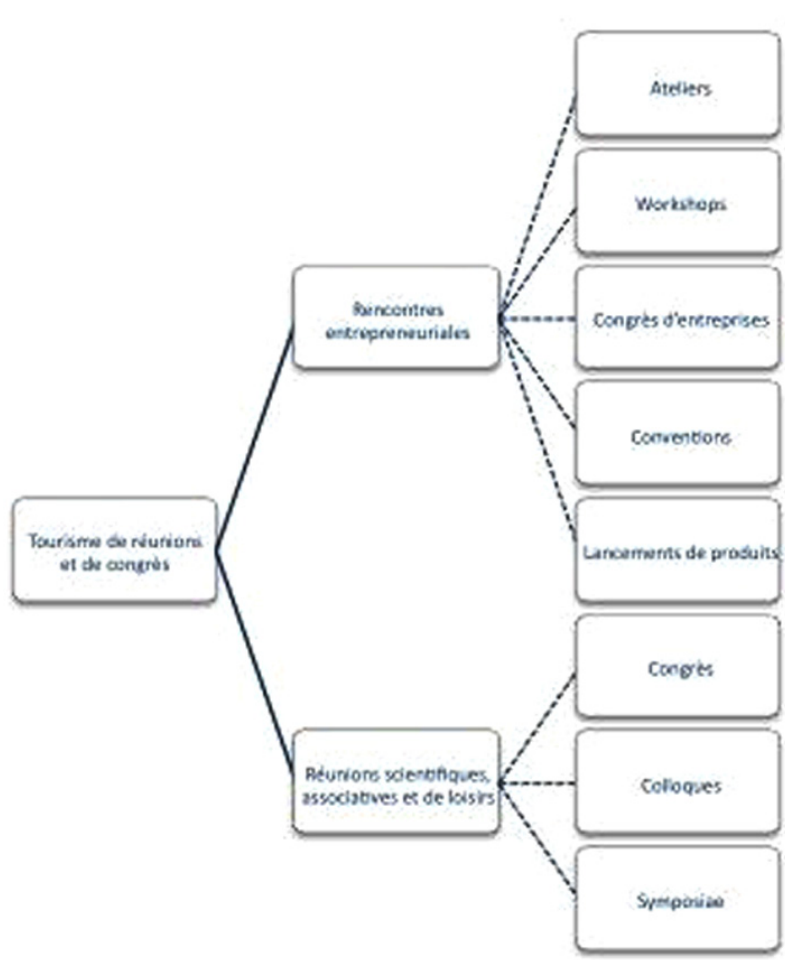

Fig. 1. Typologie des manifestations du tourisme de réunions et de congrès.

scientifique et communicationnelle (Davezies, 2007) à l'échelle continentale et nationale dans les zones les plus développées, à l'échelle intra-nationale dans les capitales, les métropoles, voire les stations et villes touristiques de haut niveau (Christofle, 2003, Péloquin, 2005, UAI, 2010, Christofle, 2012). Particulièrement pour les grandes manifestations, notamment internationales, les délégués se retrouvent dans un espace spécialisé, le palais des congrès.

Le tourisme de réunions et de congrès se comprend donc en véritable système, avec un fonctionnement propre, à la fois proche et différent des autres formes touristiques et des déplacements dits d'affaires (voyages commerciaux et d'affaires, participation à des foires, salons etc.)

L'architecture du système congressuel s'articule de la manière suivante :

- une pratique touristique composite : réunion et activités associées $\rightarrow$ transmission d'informations, échange \& interaction, visites, shopping, découverte, culture...

- quatre groupes d'acteurs majeurs : organisateurs de congrès, congressistes \& accompagnants, institutionnels du tourisme et collectivités territoriales, collaborateurs des équipements d'accueil de réunions et fournisseurs de biens et de services liés aux meetings.

- deux principales échelles de territoires : ville d'accueil et équipement de congrès (centre et palais de congrès).

Dans le cadre conceptuel posé par le Groupe de recherche Interfaces (dont Christofle, 2008), le tourisme de réunions et de congrès est envisagé comme un système

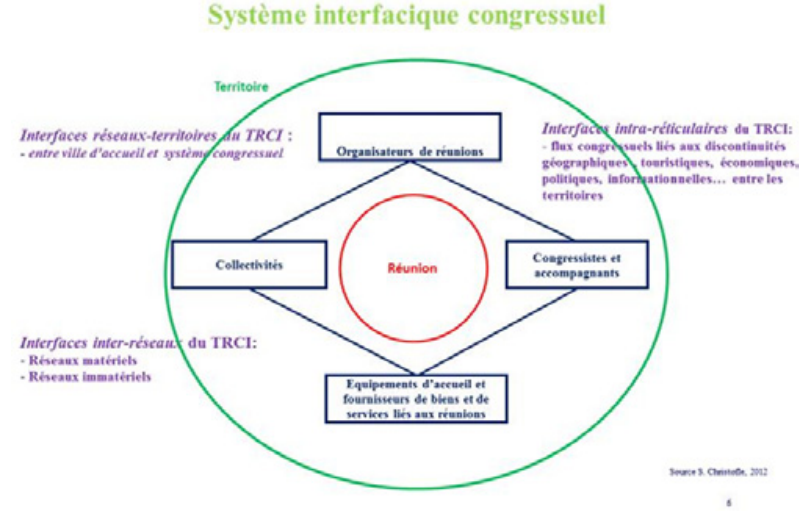

Fig. 2. Le système interfacique congressuel.

interfacique (cf. Figure 2). C'est une activité qui met en relation différents systèmes territoriaux et la sélection $\mathrm{du}$ lieu de congrès est la conséquence de régulations exercées par les centres de ces systèmes territoriaux reliés. La ville d'accueil de réunions est un espace qui procède parallèlement de complémentarités (coopération interurbaine ... ) mais aussi de différences, voire de tensions avec les autres espaces potentiels de réception de congrès (compétition, concurrence). C'est un poste de contact entre, d'une part, l'activité congressuelle, fonction touristique et communicationnelle élitaire fonctionnant en réseau, d'autre part les équipements dédiés (centres et palais des congrès) et enfin les différents territoires d'interconnexion et ce, à plusieurs échelles (quartier, localité, pays...). La régulation et la valorisation des flux congressuels \& touristiques est portée par des groupes d'acteurs socioéconomiques et institutionnels, et les principaux échanges entre les territoires sont les entrées et sorties du système. Son organisation interne privilégie la sélection et la mise en valeur d'échanges variés : transmission d'informations, rencontres, discussions, mise en place et consolidation de réseaux interpersonnels, relations économique, culturelles, sociales avec le territoire d'accueil etc. Le système congressuel est un système ouvert, caractérisé par d'intenses relations avec son environnement ; c'est un système localisé qui se compose d'interactions entre les lieux et les sociétés. Il opère un ordonnancement entre les territoires qui possèdent et valorisent une interface congressuelle (présence d'un palais des congrès, attraction de flux de congressistes et d'accompagnants...) ou qui sont localisés dans des zones à forte dynamique congressuelle (les lieux «in»), et ceux qui n'ont pas ou peu accès à l'interface congressuel (les lieux «out») - Groupe de recherches Interfaces (2008). En effet, le tourisme de réunions et de congrès, notamment international, est issu des besoins de rencontre entre agents des fonctions urbaines et métropolitaines de haut niveau (culture, finances, économie, recherche et développement...) et nécessite des aménités touristiques/paratouristiques haut de gamme (transport, accueil, hébergement, animation...) ; plus particulièrement, le tourisme de réunions et de congrès s'appuie sur l'équipement de congrès de grande taille et de qualité. Par conséquent, la prise en compte de l'activité congressuelle par les acteurs, qui se traduit par la construction, l'agrandissement, la réhabilitation, la 
labellisation, la mise à niveau technologique constante des équipements spécialisés d'accueil de congressistes, participe à l'aménagement ainsi qu'à la hiérarchisation des lieux.

La réunion (colloque, congrès, convention etc. cf. Christofle, Fabry, Morschel, 2010) est l'interface immatérielle du système congressuel, consommée au fur et à mesure de sa production. Le tourisme de réunions et de congrès développe d'abord des interfaces intraréticulaires, notamment dans sa branche internationale. En effet, l'activité congressuelle est étroitement liée à la mondialisation et à la multiplication générale des flux et se fonde sur des ruptures à la fois géographiques $\&$ touristiques (mobilités), économiques et politiques (pouvoir d'achat, visas etc.) et informationnelles. Les disjonctions géographiques, économiques et politiques concernent particulièrement l'accessibilité des lieux, les facilités financières et les libertés politiques de déplacement, qui restent de nos jours réparties inégalement sur la planète, privilégiant les mobilités, notamment touristiques, dans et entre les espaces très développés (OMT, 2012). Le tourisme de réunions et de congrès se nourrit parallèlement des discontinuités structurelles et fonctionnelles des réseaux de conception et de distribution de l'information et de celles inhérentes au système urbain-métropolitain mondialisé, qui veut que l'information neuve, créatrice, voire stratégique a de fortes tendances à se localiser et se partager à partir de et dans les espaces-nœuds de la mondialisation (Sassen, 2001).

Dans le même temps, se manifestent les interfaces inter-réseaux du système congressuel. En effet, pour que la sélection du lieu de réunion soit effectuée (après candidatures des responsables locaux des associations ou décision des entrepreneurs), que les congressistes puissent rejoindre le lieu en question, soient hébergés, nourris, divertis, que la réunion puisse se tenir... doivent être mis en contact des réseaux multiples, matériels : de transports, d'hébergement, techniques, technologiques..., et immatériels : entrepreneuriaux, associatifs, institutionnels, scientifiques. .

L'équipement spécialisé, le palais des congrès, porte d'entrée majeure, interface matérielle du système congressuel, maximise alors les interfaces réseaux-territoires, concentrant les flux de personnes et d'informations dans des territoires privilégiés : stations touristiques prestigieuses et métropoles des pays développés. Ces localités se révèlent les interfaces spatiales entre le local et le global, valorisant rencontres et échanges de toutes natures et de toutes tailles... avec, à chaque fois, des conséquences sur les habitants qui sont en lien avec ces interfaces multiscalaires. En effet, le système interfacique congressuel crée ou multiplie des effets territoriaux synergétiques de différentes natures : concentration, concurrence, coopération, développement... et influe sur les dynamiques territoriales. Cette forme de mobilité touristique se développe particulièrement à l'heure de la mondialisation car elle se nourrit de la facilitation accrue des déplacements internationaux, des besoins croissants de connaissances de haut niveau, de la nécessité toujours renouvelée de mise en place et de consolidation de réseaux en face-à-face, de l'attractivité des destinations touristiques. Le tourisme de réunions et de congrès, en croissance

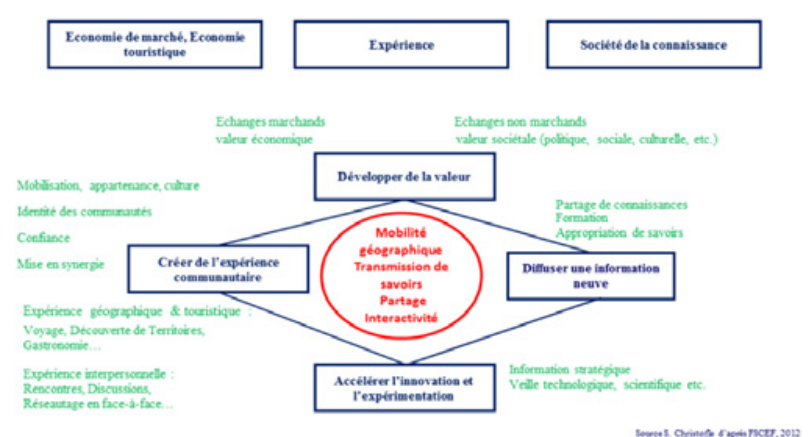

Fig. 3. Principaux effets d'interfaçage induits du Tourisme de réunions et de congrès.

quasi-continue depuis plusieurs décennies (UAI), est ainsi perçu comme une activité qui valorise le territoire d'accueil sous de multiples formes : économie, affaires, tourisme, communication, innovation (Christofle, 1997, Habibullah, Rex and Lyndon, 2005).

La modélisation des principaux effets induits par l'interfaçage permet maintenant de poursuivre l'exploration du système.

\section{Effets majeurs}

La réunion, au centre du schéma (cf. Fig. 3 Effets interfaciques congressuels majeurs, ci-dessus) engendre un déplacement géographique, est source de transmission de savoirs, de partage et d'interactivité entre les participants au congrès.

Les effets d'interfaces majeurs induits par la participation à un congrès, colloque etc. sont au nombre de quatre :

- la diffusion et l'appropriation d'une information neuve

- l'accélération de l'innovation et de l'expérimentation

- la création de l'expérience communautaire

- le développement de la valeur.

La diffusion et l'appropriation d'une information neuve est le premier effet, le plus attendu rationnellement. En effet, une des finalités majeures de la mobilité congressuelle est l'acquisition, le partage de connaissances voire la formation. Dans les congrès se transmettent traditionnellement des informations récentes voire inédites, des résultats, des réflexions abouties qui ont pour objet de faire avancer la science, les savoirs, de donner des indications sur l'état d'institutions ou d'entreprises voire de révéler des données stratégiques. L'activité de réunions peut ainsi s'insérer en amont du processus de circulation de l'information. En effet, «il est de plus en plus communément admis que les savoirs doivent être gérés comme les ressources matérielles, l'idée étant même de plus en plus acquise que c'est là que réside désormais la clé de la compétitivité » (Tarondeau, 1998, Mbengue 2004). L'information intervient par conséquent dans les circuits de l'innovation des entreprises, des laboratoires de recherche voire des réseaux qui relient industries, services et recherche scientifique, circuits participant à l'interactivité informationnelle. Au sein des organismes privés, l'information doit atteindre, 
au-delà des personnels affectés à la recherche et au développement, les services de design, publicité, marketing et secteur commercial qui ont leur rôle à jouer dans la bonne marche de l'organisation au sein du système global de l'innovation, personnels qui se retrouvent à l'occasion de réunions d'entreprises, conventions... De multiples séminaires et journées d'études sont organisés et permettent d'apprécier les changements dans le savoir-faire, liés également aux processus d'innovation, dans les fonctions, les techniques et l'organisation des systèmes de production, associés aux changements technologiques. A ces éléments de développement s'ajoutent la complexité croissante des milieux financiers, nationaux et internationaux, qui requièrent des informations de plus en plus précises, l'intégration internationale de la production et de la consommation, la réglementation accrue dans la plupart des pays développés, associés à l'accroissement de l'intensité et des interactions entre organismes qui influent sur le nombre des réunions. Ce faisant, l'information originale dispensée, discutée, agrémentée, amendée peut permettre de fonder ou d'accélérer l'innovation et l'expérimentation en renforçant la veille technique, technologique ou scientifique (résultats scientifiques, dépôts de brevets etc.) Parallèlement, la présence à un congrès, un colloque etc. crée de l'expérience communautaire. En effet, les participants sont liés par un ou des intérêts communs, particulièrement l'appartenance à une même entité, laboratoire, association socio-professionnelle ou de loisirs ${ }^{2}$, organisation gouvernementale ou non gouvernementale ${ }^{3}$, entreprise, monde académique et universitaire... La mobilisation des membres, d'autant plus quand existe une véritable culture commune, permet de mettre en synergie les participants, crée de la confiance dans le collectif et entraîne un sens communautaire consolidé. En sus de la réunion strictosensu, les moments partagés, qu'ils soient formels : pausescafé, repas, dîner de gala, ou informels : discussions, rencontres, sorties, visites etc. sont créateurs d'expérience et de moments parfois mémorables, qui entretiennent et valorisent le lien. Se mettent en place ou se raffermissent des réseaux interpersonnels autorisant de multiples échanges, interactions et interrelations durant la réunion et bien audelà, réseaux d'amitiés, professionnels etc. Parallèlement, les mobilités congressuelles peuvent se révéler de véritables expériences géographiques et touristiques via le voyage (notamment lors des congrès internationaux), des découvertes de territoires, de gastronomie, les périodes de pré et post congrès, l'envie de revenir pour un séjour de loisirs ... Tous ces échanges développent donc, in fine, de la valeur, qu'elle soit marchande ou sociétale (politique, sociale, culturelle...). L'activité congressuelle est ainsi une forme touristique richement interfacée à l'échelle des sphères économico-touristique, expérientielle et sociétale.

Après cette caractérisation du tourisme de réunions et de congrès et du système interfacique congressuel, il va être analysé quel est le fonctionnement dynamique des différentes chaînes interfaciques générées.

\footnotetext{
${ }^{2}$ Association nationale des Elus de la Montagne, Ordre des notaires, Association internationale de littérature comparée, Fédération Internationale pour la Musique Chorale... sont des exemples de structures organisant régulièrement des congrès.

${ }^{3}$ Croix-Rouge, Union européenne...
}

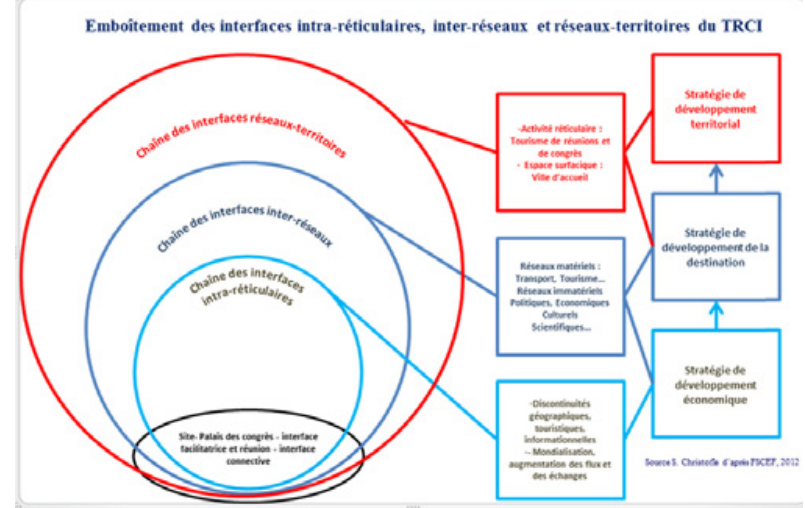

Fig. 4. Emboîtement des interfaces congressuelles.

\section{Dynamique interfacique du système congressuel}

En effet, (Groupe de recherches Interfaces, 2008) les systèmes interfacés sont modifiés par l'existence même de l'interface, ce qui entraîne des conséquences sur le plan économique, social, culturel, etc. A partir des interfaces originelles du système étudié, d'une part la réunion, interface immatérielle et connective et d'autre part, le site de réunion-palais des congrès, interface matérielle et facilitatrice, se mettent en place des chaînes d'interfaces majeures, intra-réticulaires, interréseaux, réseaux-territoires (cf. Figure 4).

La chaîne des interfaces intra-réticulaires congressuelles est liée à la mondialisation tant économique que culturelle, informationnelle, touristique etc. qui peut accentuer, d'une part, des différentiels entre les territoires et, d'autre part, multiplie les besoins d'échanges et les flux de toutes formes et de toutes tailles entre les espaces. Ces éléments entraînent une augmentation des rencontres et une concentration de celles-ci dans des lieux privilégiés, essentiellement les grands pays développés, qui profitent des ruptures touristiques et métropolitaines entre les zones du monde. Ces parties prenantes privées et publiques de la chaîne des interfaces intra-réticulaires se situent essentiellement dans l'optique d'un développement économique de leurs structures.

La chaîne des interfaces inter-réseaux congressuelles met en contact les multiples réseaux inhérents au tourisme de réunions et de congrès. D'une part, se trouvent les réseaux matériels, permettant la réalisation de l'activité transport, logistique, hébergement, restauration, technologies, animation... et, d'autre part, les réseaux immatériels, source des besoins de rencontres et porteurs de projets congressuels : représentants locaux des organismes qui sont à l'initiative des congrès (entreprises, laboratoires, associations...). Les nœuds des réseaux matériels (hôtels, gares, aéroports...) concourent à la masse critique d'aménités nécessaires en quantité et en qualité pour que la candidature de la ville d'accueil de congrès international soit examinée par les instances dirigeantes de l'entreprise ou de l'association génératrice de congrès, candidature portée, pour les réunions associatives et scientifiques, par le représentant local de l'entité en question (laboratoire, 
fédération...), nœud du réseau immatériel. Pour les réunions entrepreneuriales le portage territorial de projet est moins systématique mais, dans les deux cas, le choix final du lieu de réunion résulte de la balance entre les apports des différents acteurs des réseaux matériels et immatériels dans le cadre d'une stratégie de développement de la destination.

Enfin, la chaîne des interfaces réseaux-territoires concerne la liaison entre le fonctionnement réticulaire de l'activité congressuelle et l'espace surfacique d'accueil de congrès, la ville. Le système congressuel se fonde sur un panel important de villes pour se développer car les congrès se localisent en un lieu différent à chaque édition. Au-delà des places fortes traditionnelles de congrès (capitales et grandes métropoles des espaces très développés), il est donc possible pour un territoire moins privilégié de se positionner comme lieu potentiel de congrès en mettant en place des stratégies territoriales. Ces stratégies se matérialisent essentiellement en des actions d'aménagement, création de palais des congrès, interfaces indispensable de développement de l'activité dans la cité, confortées par des politiques plus générales d'internationalisation et de touristification des lieux, soutenues par des campagnes de marketing territorial. Le tourisme de réunions et de congrès est donc perçu en ce cas par les décideurs politiques et institutionnels comme une composante importante dans des stratégies de développement territorial, interface local-global qui a pour fonction de capter et de canaliser des flux de congressistes \& d'accompagnants (Groupe de recherches Interfaces, 2008) et d'assurer des retombées économiques et médiatiques à la ville d'accueil de congrès.

Le système touristique congressuel, interpénétrant les mondes académique, économique, d'affaires et associatif se révèle très prometteur pour la recherche interfacique. En effet, la chaîne des interfaces congressuelles, tant immatérielles que matérielles, intra-réticulaires, inter réseaux et réseaux-territoires entraînent de multiples effets, informationnels, expérientiels, d'innovation et de valorisation qui irriguent, dynamisent les territoires interfacés et restent en bonne partie encore à explorer...

\section{Références}

Boyer M., 2002, «Comment étudier le tourisme ?» in Ethnologie française, Tome XXVII, pp.393-404

Christofle, 1997, Tourisme de réunions et politiques urbaines, le cas du Corum de Montpellier, Thèse de doctorat de géographie, Université Montpellier III, 767 p., 1997.

Christofle, 2003, «Congrès internationaux, réseaux de villes et mondialisation $\gg N E T C O M$, vol. $17, \mathrm{n}^{\circ} 1-2$ pp. 71-80.

Christofle S. Massiera B. (2009) «Industrie de l'événement, congrès internationaux et stratégies métropolitaines 》 Chapitre dans Volume 1 Coprésence à l'œuvre, ouvrage sous la direction de Jean Pierre Lemasson et Philippe Violier. Édition Téoros. Presses université du Québec pp. 220-230.
Christofle S. Fabry N. Morschel J. (2010a) «Paris et le tourisme de réunions et de congrès, une métropole en compétition », Communication (2010) et Actes du colloque international (à paraître) : Paris, Tourisme et Métropolisation, Paris.

Christofle S. (2010b) «Interfaces Villes, Tourisme de réunions et de congrès, Palais des Congrès », chapitre dans Géographie des interfaces, ouvrage coordonné par P. Allard et S. Pérez, Ed. Quae, 12 p.

Christofle S. (2011) «Une thématique émergente dans le champ de la géographie du tourisme : le tourisme de réunions et de congrès $»$, Colloque national sur la Recherche et l'Enseignement Supérieur en Tourisme ASTRES, Toulouse, 23-24 mai 2011.

Christofle S. (2012), Communication « Comment conserver et développer un leadership territorial en tourisme de réunions et de congrès ? Le cas de la Côte d'Azur», Colloque national sur la Recherche et l'Enseignement Supérieur en Tourisme - ASTRES, Nice, 2-3 avril 2012.

Davezies L., Compétition internationale et intégration subnationale : des effets territoriaux contrastés, L'Économie Politique 2007/1, n³3, p. 53-66.

Davidson, R. (2003). Adding pleasure to business : Conventions and tourism. Journal of Convention and Exhibition Management, 5(1), 29-39.

Groupe de recherches «Interfaces» (2008) (Emsellem K., Allard P., Christofle S., Ferrier JP, Fusco G., Gay JC., Kaddouri L., Lampin C., Lavaud-Letilleul V., Pérez S.) animé par L. CHAPELON, UMR 6012 ESPACE «L'interface : contribution à l'analyse de l'espace géographique », L'Espace Géographique, 2008-3, pp. 193-207.

Larquet V. Foires, salons, Congrès et Evènements de France- FSCEF, « Réinventer les foires, salons et congrès - Etat des lieux de la réflexion FSCEF », Communication à la Journée d'études «Foires, Salons \& Congrès dans la Cité », Toulouse, avril 2012.

Habibullah K., Rex S. T. and Lyndon C., "Tourism and Trade : Cointegration and Granger Causality", Journal of Travel Research 2005; 44; 171

Mbengue A., Management des savoirs, Revue française de gestion 2004/2, $\mathrm{n}^{\circ} 149$, p. 13-31.

Meyer C., 2005, «Le tourisme : essai de définition », Revue Management et avenir 2005/1, n 3 , pp. 7-25.

Peloquin C., 2005, «Regards sur le tourisme de congrès », Le Globe-veilleur, réseau de veille en Tourisme, UQAM, Canada. http:// veilletourisme.ca/2005/06/15/regard-sur-letourisme-de-congres/

Pine J. \& Gilmore J.H. (1999) "The Experience Economy : Work is Theatre \& Every Business a Stage", Harvard Business Press, 254 pages.

Sassen S. (2001) : The global city : New York, London, Tokyo Princeton, N.J. : Princeton University Press. 2d ed.

Stock M. (coord.), 2003, Le tourisme, acteurs, lieux et enjeux, Belin Sup Géographie, Paris.

Tarondeau J. C., Le management des savoirs, Que-sais-je ?, PUF, Paris, 1998.

U.A.I Union des Associations Internationales, Meetings statistics report for the year 2011, Bruxelles, 67 pages. 